\title{
TIGHT FIBRED KNOTS WITHOUT L-SPACE SURGERIES
}

\author{
FILIP MISEV \\ Max Planck Institute for Mathematics, Bonn, Germany \\ e-mail:fmisev@mpim-bonn.mpg.de \\ and GILBERTO SPANO \\ LMNO, Université de Caen-Normandie, Caen, France \\ e-mails: gilbertospano.math@gmail.com,gilberto.spano@unicaen.fr
}

(Received 8 September 2020; revised 15 October 2020; accepted 22 October 2020; first published online 24 November 2020)

\begin{abstract}
We show that there exist infinitely many knots of every fixed genus $g \geqslant 2$ which do not admit surgery to an L-space, despite resembling algebraic knots and L-space knots in general: they are algebraically concordant to the torus knot $T(2,2 g+1)$ of the same genus and they are fibred and strongly quasipositive.
\end{abstract}

2020 Mathematics Subject Classification. Primary 57K10; Secondary 57K18

1. Introduction and statement of result. Algebraic knots, which include torus knots, are L-space knots: they admit Dehn surgeries to L-spaces, certain 3-manifolds generalising lens spaces which are defined in terms of Heegaard Floer homology [7].

The first author recently described a method to construct infinite families of knots of any fixed genus $g \geqslant 2$ which all have the same Seifert form as the torus knot $T(2,2 g+1)$ of the same genus, and which are all fibred, hyperbolic and strongly quasipositive. Besides all the classical knot invariants given by the Seifert form, such as the Alexander polynomial, Alexander module, knot signature, Levine-Tristram signatures, the homological monodromy (in summary, the algebraic concordance class), further invariants such as the $\tau$ and $s$ concordance invariants from Heegaard Floer and Khovanov homology fail to distinguish these knots from the $T(2,2 g+1)$ torus knot (and from each other).

This is described in the article [10], where a specific family of pairwise distinct knots $K_{g, n}, n \in \mathbb{N}$, with these properties is constructed for every fixed genus $g \geqslant 2$. Figure 1 shows a diagram of the simplest non-trivial example, $K_{2,1}$.

Here we show that none of the $K_{g, n}$ is an L-space knot (except $K_{g, 0}$, which is the torus knot $T(2,2 g+1)$ by construction). This implies our main result:

THEOREM 1. For every integer $g \geqslant 2$, there exists an infinite family of pairwise distinct genus $g$ knots $K_{g, n}, n \in \mathbb{N}$, with the following properties.

(1) $K_{g, n}$ is algebraically concordant to the torus knot $T(2,2 g+1)$

(2) $K_{g, n}$ is fibred, hyperbolic and strongly quasipositive

(3) $K_{g, n}$ does not admit any nontrivial Dehn surgery to a Heegaard Floer L-space

We show in fact that the knots $K_{g, n}$ constructed in [10] do not have the same knot Floer homology as $T(2,2 g+1)$. 


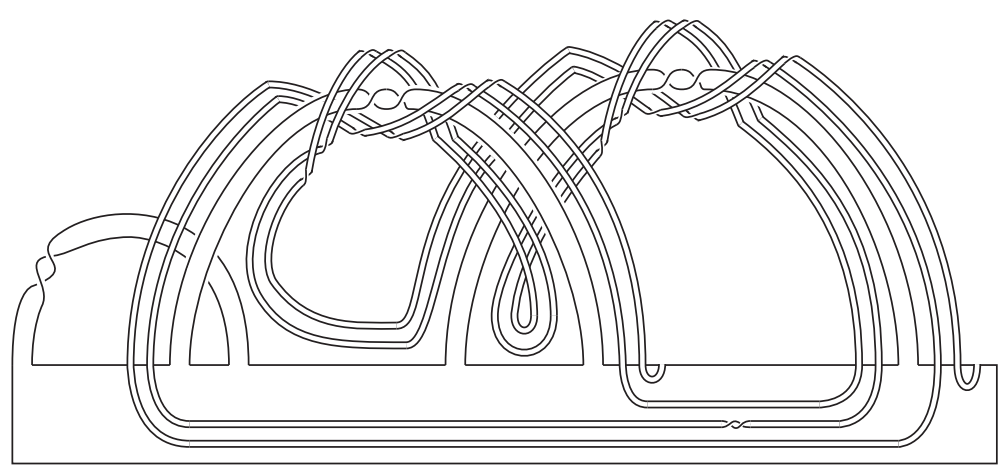

Figure 1. The knot $K_{2,1}$, as a plumbing of four Hopf bands.

It is also worth pointing out that each knot $K_{g, n} \#-T(2,2 g+1)$, for $n \geqslant 1$ and $g \geqslant 2$, is a candidate for being a counterexample to the Slice-Ribbon conjecture; compare the discussion in [10].

Below we briefly introduce the notions of L-spaces, L-space knots, quasipositivity and fibredness and relate our result to recent work by Boileau, Boyer and Gordon on the subject of L-space knots. Section 2 contains a description of the fibred knots $K_{g, n}$ via their monodromy (taken from [10]) and collects the main ingredients from Heegaard Floer theory and Lagrangian Floer homology used in the proof of our result, which is given in Section 3.

1.1. L-spaces, quasipositivity and fibredness. L-spaces are named after lens spaces, three-dimensional manifolds formed by glueing two solid tori along their boundaries. By definition, a closed 3-manifold $M$ is an $L$-space if it is a rational homology sphere, that is, $H_{*}(M, \mathbb{Q}) \cong H_{*}\left(S^{3}, \mathbb{Q}\right)$, and its Heegaard Floer homology has the smallest possible rank: $\operatorname{rk} \widehat{\mathrm{HF}}(M)=\left|H_{1}(M, \mathbb{Z})\right|$. Every lens space (except $S^{1} \times S^{2}$, which fails to be a rational homology sphere) is in fact an L-space. More generally, 3-manifolds with finite fundamental group (the manifolds with elliptic geometry, certain Seifert fibred manifolds) are known to be L-spaces [16, Proposition 2.3].

A knot $K \subset S^{3}$ is an $L$-space knot if some positive integral Dehn surgery on $K$ yields an L-space. Basic examples include the torus knots and the Berge knots, since they admit lens space surgeries; see $[\mathbf{1}, \mathbf{6}, \mathbf{1 2}]$. In addition, all algebraic knots (the connected links of plane curve singularities, which include all positive torus knots) are L-space knots. This follows from a theorem of Hedden stating that certain cables of L-space knots are again L-space knots [7, Theorem 1.10], combined with the classical description of algebraic knots as iterated cables of torus knots, involving the Puiseux inequalities (see, for example [3]).

By work of Ghiggini [5] and Ni [13, Corollary 1.3], [14], all L-space knots are known to be fibred: they arise as the bindings of open book decompositions of $S^{3}$. In addition, Hedden proved that the open book associated to an L-space knot (or to its mirror) supports the tight contact structure of $S^{3}$, under Giroux' correspondence (see [8, Theorem 1.2, Proposition 2.1] and Ozsváth-Szabó [16, Corollary 1.6]). In summary, L-space knots are tight fibred knots.

Besides Dehn surgery, another important construction of 3-manifolds, starting from a knot $K \subset S^{3}$, is given by branched covering of $S^{3}$ branched along $K$. The $n$-fold cyclic branched covering, branched along $K$, is denoted $\Sigma_{n}(K)$. For example, if $K$ is an alternating knot, $\Sigma_{2}(K)$ is always an L-space [17]. 
Recently, Boileau et al. [2] considered L-space knots $K \subset S^{3}$ with the additional property that $\Sigma_{n}(K)$ is also an L-space for some $n \in \mathbb{N}, n \geqslant 2$. They deduced strong restrictions on such knots. In order to state their results, we briefly recall the notion of strongly quasipositive knots, which were introduced and first studied by Rudolph in the 80s [19]. Let the symbols $\sigma_{1}, \sigma_{2}, \ldots, \sigma_{n-1}$ denote the standard positive generators of the braid group on $n$ strands, $\sigma_{i}$ corresponding to the braid in which the $i$-th strand crosses over the $(i+1)$-st strand in the direction of the braid's orientation (and no other crossings). A braid $\beta$ is called strongly quasipositive if it can be written as a product of conjugates of the $\sigma_{i}$ :

$$
\beta=\prod_{j=1}^{d} w_{j} \sigma_{n_{j}} w_{j}^{-1},
$$

where $d \in \mathbb{N}, n_{1}, \ldots, n_{d} \in\{1, \ldots, n-1\}$ and the conjugating words $w_{j}$ are of the special form

$$
w_{j}=\sigma_{n_{j}-k} \cdots \sigma_{n_{j}-1}
$$

for some $k$ (depending on $j$ ). Accordingly, such braids are called strongly quasipositive. Hedden showed that a fibred knot is tight if and only if it is strongly quasipositive [8]. Fibredness is important here: there do exist non-fibred strongly quasipositive knots.

THEOREM 2 (Boileau et al. [2]). Let $K$ be a strongly quasipositive knot with monic Alexander polynomial. Then

(1) $\Sigma_{n}(K)$ is not an L-space for $n \geqslant 6$.

(2) If $\Sigma_{n}(K)$ is an L-space for $2 \leqslant n \leqslant 5$, then $K$ has maximal signature and its Alexander polynomial is a product of cyclotomic polynomials.

COROLlary 1 (Boileau et al. [2]). Let $K$ be an L-space knot such that $\Sigma_{n}(K)$ is an L-space for some $n$. Then

(1) $n \geqslant 4$ implies that $K$ is the trefoil knot.

(2) $n=3$ implies that $K$ is either the trefoil knot or its Alexander polynomial is equal to $t^{4}-t^{3}+t^{2}-t+1$, the Alexander polynomial of the cinquefoil knot. If it is neither the trefoil nor the cinquefoil, it is a hyperbolic knot.

These results suggest that the two properties, admitting an L-space surgery and admitting an L-space branched cover are orthogonal in the sense that only few knots seem to satisfy both properties simultaneously. When applied to genus $g$ knots $K_{g, n}$ satisfying the properties of Theorem 1 (and described in the next section), the above Corollary 1 implies that $\Sigma_{m}\left(K_{g, n}\right)$ is not an L-space for $m, g \geqslant 3$ and $n \neq 0$ :

Corollary 2. Fix $g \geqslant 3$ and let $K_{g, n}$ denote the genus $g$ knot described below. If $m \geqslant 3$ and $n \neq 0$, the $m$-fold cyclic branched cover $\Sigma_{m}\left(K_{g, n}\right)$ of $S^{3}$, branched along $K_{g, n}$ is not an L-space.

For $g=2$, the knots $K_{g, n}$ have the same Alexander polynomial as the cinquefoil knot $T(2,5)$. The following (open) question was brought to our attention by Ken Baker, Michel Boileau, Marco Golla and Arunima Ray, independently.

QUESTION. Is the double branched cover $\Sigma_{2}\left(K_{g, n}\right)$ an L-space for any of the knots $K_{g, n}$, $n \neq 0$ (mentioned in Theorem 1 and described below)? Is $\Sigma_{3}\left(K_{2, n}\right)$ an L-space? 


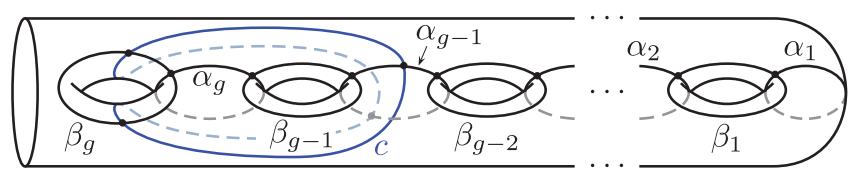

Figure 2. The surface $S$ of genus $g$ and one boundary circle with the twist curves $\alpha_{i}, \beta_{j}$.

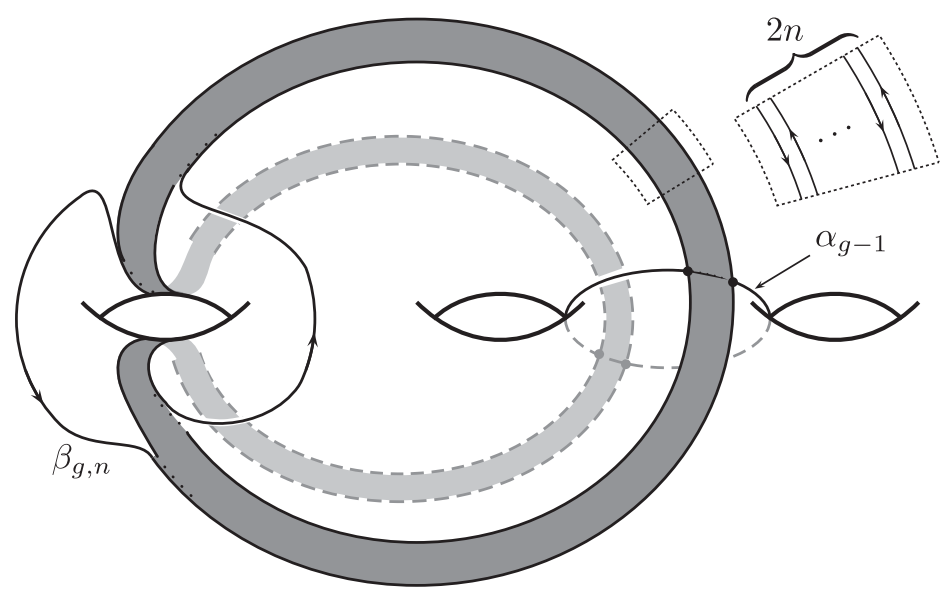

Figure 3. The curve $\beta_{g, n}=t_{c}^{n}\left(\beta_{g}\right)$ wraps $n$-times around the curve $c$ in both directions which gives a total of $2 n$ strands parallel to $c$ (represented by the shaded band). $\alpha_{g-1}$ and $\beta_{g, n}$ intersect in $4 n$ points.

\section{Monodromies, L-spaces and exact triangles.}

2.1. The monodromy of the fibred knots $K_{g, n}$. Let us recall the construction of the knots $K_{g, n}$ from the article [10]. Throughout, we fix an integer $g \geqslant 2$, the genus of the knots to be constructed. The fibred knots $K_{g, n}$ are given in terms of their monodromies: $K_{g, n}$ has a genus $g$ fibre surface $S_{n}$. Since the topological type of $S_{n} \subset S^{3}$ does not depend on $n$, we can identify $S_{n}$ with a fixed abstract (non-embedded) surface $S$ and consider the monodromy of $K_{g, n}$ as a mapping class $\varphi_{n}: S \rightarrow S$. Given a simple closed curve $\gamma \subset S$, we denote $t_{\gamma}: S \rightarrow S$ the right Dehn twist on $\gamma$. Using this notation, the monodromy $\varphi_{n}, n \in \mathbb{N}$, is defined as the following composition of Dehn twists:

$$
\varphi_{n}:=\left(t_{\beta_{g, n}} \circ t_{\beta_{g-1}} \circ \ldots \circ t_{\beta_{1}}\right) \circ\left(t_{\alpha_{g}} \circ \ldots \circ t_{\alpha_{1}}\right)
$$

where

$$
\beta_{g, n}:=t_{c}^{n}\left(\beta_{g}\right)
$$

and $\alpha_{i}, \beta_{j}$ and $c$ are the simple closed curves shown in Figure 2. The curve $c$, shown in blue, is the boundary of a neighbourhood of $\alpha_{g} \cup \beta_{g-1}$ in $S$. In particular, it is nullhomologous, intersects $\beta_{g}$ and $\alpha_{g-1}$ in exactly two points each and does not intersect any of the remaining curves.

It follows that $\beta_{g, n} \cap \alpha_{g-1}$ consists of $4 n$ points (see Figure 3). Since $\beta_{g}$ and $\alpha_{g}$ intersect in exactly one point and $\alpha_{g}, c$ are disjoint, $\beta_{g, n} \cap \alpha_{g}$ is a singleton. Moreover, all pairs of curves involved in the construction realise their minimal geometric intersection number in their homotopy classes. This is clear whenever two curves are disjoint or intersect transversely in a single point. For the remaining cases, use the bigon criterion 
[4, Proposition 1.7]. In particular, this applies to the curves $\beta_{g, n}$ and $\alpha_{g-1}$, whose minimal geometric intersection number $\iota\left(\alpha_{g-1}, \beta_{g, n}\right)=\#\left(\alpha_{g-1} \cap \beta_{g, n}\right)=4 n$ is used in the proof of our Theorem 1. These two curves are represented in Figure 3 (the shaded band represents $2 n$ parallel strands with alternating orientations which are part of $\beta_{g, n}$ ).

2.2. Floer homology of L-space knots. The proof of our result, Theorem 1, relies on the following theorem by Ozsváth-Szabó, which implies that the knot Floer homology groups of an L-space knot are at most one-dimensional in each Alexander degree.

THEOREM 3 (Ozsváth-Szabó, Theorem 1.2 in [16]). Let $K \subset S^{3}$ be a knot which admits a positive integral L-space surgery. Then, there exists a sequence of integers $n_{0}=0<n_{1}<$ $\ldots<n_{k}$ such that

$$
\widehat{\operatorname{HFK}}_{d}\left(S^{3}, K ; j\right) \cong \begin{cases}\mathbb{Z} & \text { if }(j, d)=\left( \pm n_{i}, \delta_{i}\right), \text { for some } i \\ 0 & \text { otherwise, }\end{cases}
$$

where the supporting dimensions $\delta_{i}$ only depend on the $n_{i}$, according to the recursive formula

$$
\delta_{i}= \begin{cases}0 & \text { if } i=k \\ \delta_{i+1}-2\left(n_{i+1}-n_{i}\right)+1 & \text { if } k-i \text { is odd } \\ \delta_{i+1}-1 & \text { if } k-i>0 \text { is even }\end{cases}
$$

We make use of two exact triangles in knot Floer homology and in Lagrangian Floer homology to bound the rank of the knot Floer homology groups of our knots $K_{g, n}$ from below. Since the $K_{g, n}$ are all fibred of genus $g$, their knot Floer homology groups in Alexander degree $\pm g$ have rank one. To prove that $K_{g, n}$ is not L-space for $n>0$ we will show that

$$
\operatorname{rk} \widehat{\operatorname{HFK}}\left(S^{3}, K_{g, n} ;-g+1\right)>1,
$$

which contradicts the condition of the last theorem. Note also that it does not suffice to consider the coefficients of the Alexander polynomial of the genus $g$ knot $K_{g, n}$, since it equals the Alexander polynomial of the torus knot $T(2,2 g+1)$, whose coefficients are all \pm 1 .

2.3. Two exact triangles in knot Floer homology and in Lagrangian Floer homology. Recall that the monodromies of the knots $K_{g, n}$ only differ by a single Dehn twist. In order to estimate their Floer homology ranks, we wish to relate the Floer homology groups of fibred knots (in possibly distinct 3-manifolds) whose monodromies differ by a Dehn twist. This is done by realising the composition of the monodromy with a Dehn twist as a Dehn surgery and using the surgery exact sequence for Floer homology, as follows.

Let $Y$ be a closed oriented 3-manifold and let $K \subset Y$ be a genus $g$ fibred knot with associated monodromy $\varphi: S \rightarrow S$, so that, if $\mathcal{N}$ is a tubular neighbourhood of $K, Y \backslash \mathcal{N}$ is homeomorphic to the mapping torus $(S \times[0,1]) /((x, 1) \sim(\varphi(x), 0))$. An essential simple closed curve $\gamma \subset S$ can then be identified with a knot $L_{\gamma} \subset S \times\{0\} \subset Y$.

Fix $\gamma$ and assume that $L_{\gamma}$ is non-trivial. Let $K^{\prime} \subset Y^{\prime}:=Y_{-1}\left(L_{\gamma}\right)$ and, respectively, $K_{0} \subset Y_{0}:=Y_{0}\left(L_{\gamma}\right)$ be the knots determined by $K$ in the manifolds obtained via -1 and, respectively, 0 -surgery on $L_{\gamma}$. Here we use the framing of $L_{\gamma}$ induced by the surface $S$. 
For example, 0 -surgery along $L_{\gamma}$ consists in removing a solid torus neighbourhood of $L_{\gamma} \subset Y$ and gluing it back in, identifying its meridian to a parallel push-off of $\gamma$ inside of $S \times\{0\}$. With this convention, $K^{\prime}$ is also a genus $g$ fibred knot, with associated fibre $S$ and monodromy $\varphi \circ t_{\gamma}$. On the other hand, $K_{0}$ has genus at most $g-1$, since we can compress $S \subset Y_{0}$ along the curve $L_{\gamma}$ to obtain a genus $g-1$ Seifert surface $F \subset Y_{0}$ of $K_{0}$. Using this notation, $(S, \gamma, \varphi(\gamma))$ becomes a sutured Heegaard diagram for the sutured manifold $Y_{0}(F)$. This means that the sutured manifold $Y_{0}(F):=\left(Y_{0} \backslash(F \times[0,1]), \partial F \times[0,1]\right)$ is obtained from the product sutured manifold $(S \times[0,1], \partial S \times[0,1])$ by attaching a 2-handle along $\gamma \times\{0\}$ and a 2-handle along $\varphi(\gamma) \times\{1\}$ (see the Definitions 2.4 and 2.10 of [9]). Theorem 1.5 of [9] and the definition of sutured Floer homology imply that

$$
\widehat{\operatorname{HFK}}\left(Y_{0}, K_{0} ;-g+1\right) \cong \operatorname{HF}(\gamma, \varphi(\gamma)),
$$

where the latter is the Lagrangian Floer homology of $(\gamma, \varphi(\gamma))$. This is a homology whose generators of the chain complex are intersection points of $\gamma$ and $\varphi(\gamma)$ (which are assumed to be transverse; see for example [20] for the details of the definition). We recall also that if two curves $a$ and $b$ are homotopic then

$$
\operatorname{rk} \operatorname{HF}(a, b)=2
$$

and if they are not homotopic then

$$
\operatorname{rkHF}(a, b)=\iota(a, b),
$$

where $\iota(a, b)$ denotes the geometric intersection number of the curves $a$ and $b$.

LEMMA 1. Using the same notation as above, that is, $K \subset Y$ is a fibred knot of genus $g$ with monodromy $\varphi: S \rightarrow S, \gamma \subset S$ is an essential simple closed curve and $\left(Y^{\prime}, K^{\prime}\right)$ is obtained from $(Y, K)$ by $(-1)$-Dehn surgery along $\gamma \subset Y$, the following triangle is exact.

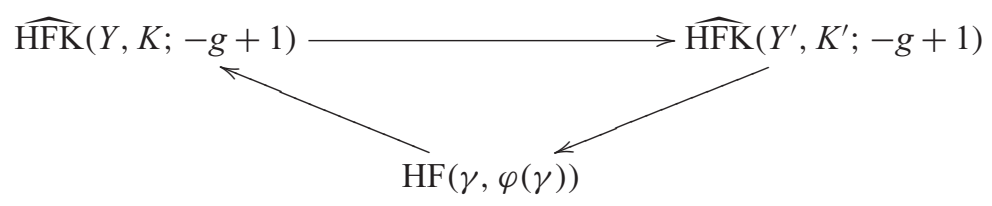

Proof. This is a direct consequence of (2.1) and Ozsváth and Szabó's exact sequence for knot Floer homology.

The second lemma we are going to use is a special case of a theorem due to Seidel. It generalises the well-known identity $\iota\left(t_{a}(b), b\right)=\iota(a, b)^{2}$ for essential simple closed curves $a, b$ on a surface [4, Proposition 3.2].

LEMMA 2 (Seidel [20]). Let $S$ be a compact oriented surface with boundary and $t_{c}$ : $S \rightarrow S$ the Dehn twist on a simple closed curve $c \subset S$. For any pair of simple closed curves $a, b \subset S$, there is an exact triangle of Lagrangian Floer cohomology groups

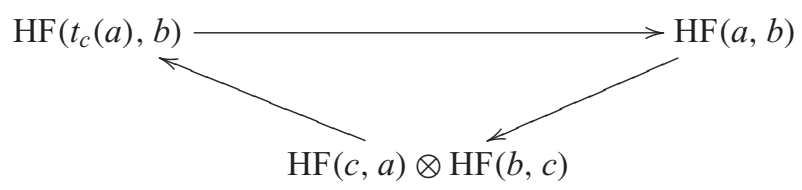




\section{Proof of the main theorem.}

Proof of Theorem 1. Let $\psi:=t_{\beta_{g-1}} \circ \ldots \circ t_{\beta_{1}} \circ t_{\alpha_{g}} \circ \ldots \circ t_{\alpha_{1}}$, so that $\varphi_{n}=t_{\beta_{g, n}} \circ \psi$. Let $(Y, K)$ be given by the open book $(S, \psi)$. That is, $K \subset Y$ is a fibred knot with monodromy $\psi: S \rightarrow S$. Note that both the 3-manifold $Y$ and the knot $K \subset Y$ are independent of $n$, since $S$ only depends on $g$ and $\psi$ is also independent of $n$. Now consider the open book $\left(S^{3}, K_{g, n}\right)$ associated to the fibred knot $K_{g, n}$, which is the knot of interest. It is obtained from $(Y, K)$ by (-1)-Dehn surgery along the curve $\beta_{g, n} \subset S \subset Y$. We can therefore apply Lemma 1 to this situation, where $\left(Y^{\prime}, K^{\prime}\right)=\left(S^{3}, K_{g, n}\right)$.

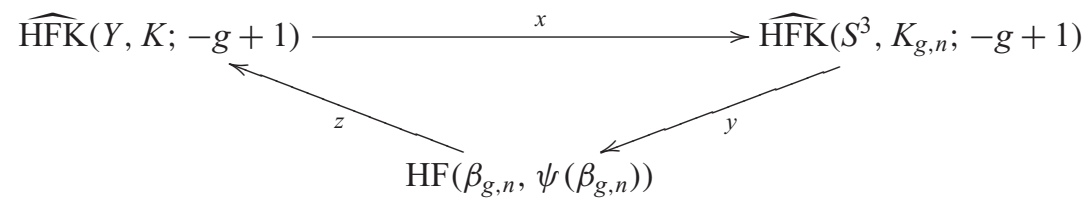

Exactness and the rank-nullity formula for $x, y$ and $z$ imply

$$
\begin{aligned}
\operatorname{rk} \widehat{\operatorname{HFK}}\left(S^{3}, K_{g, n} ;-g+1\right)= & \operatorname{dim} \operatorname{ker} y+\operatorname{dim} \operatorname{im} y \\
= & \operatorname{dim} \operatorname{im} x+\operatorname{dim} \operatorname{ker} z \\
= & \operatorname{rk} \widehat{\operatorname{HFK}}(Y, K ;-g+1)-\operatorname{dim} \operatorname{ker} x \\
& +\operatorname{rk} \operatorname{HF}\left(\beta_{g, n}, \psi\left(\beta_{g, n}\right)\right)-\operatorname{dim} \operatorname{im} z \\
\geqslant & \operatorname{rk} \operatorname{HF}\left(\beta_{g, n}, \psi\left(\beta_{g, n}\right)\right)-\operatorname{rk} \widehat{\operatorname{HFK}}(Y, K ;-g+1)
\end{aligned}
$$

To calculate the rank of $\widehat{\mathrm{HFK}}(Y, K ;-g+1)$, we apply Lemma 1 again, now taking $\left(Y^{\prime}, K^{\prime}\right)$ to be the open book obtained from $(Y, K)$ by $(-1)$-Dehn surgery along $\beta_{g, 0}=\beta_{g} \subset S \subset Y$. We obtain $Y^{\prime}=S^{3}$ and $K^{\prime}=K_{g, 0}$. The exact triangle from Lemma 1 now reads as follows.

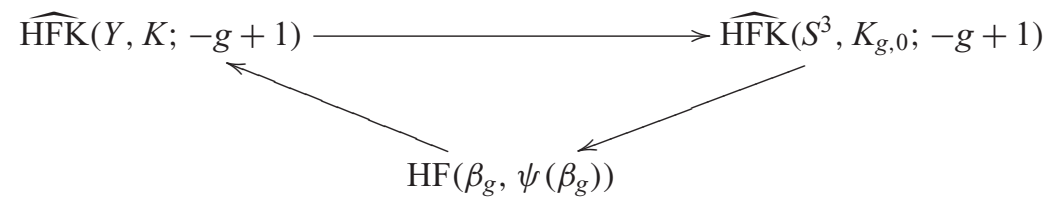

Since $K_{g, 0}=T(2,2 g+1)$, we know that rk $\widehat{\operatorname{HFK}}\left(S^{3}, K_{g, 0} ;-g+1\right)=1$. Further, the curve $\psi\left(\beta_{g}\right)=t_{\beta_{g-1}}\left(t_{\alpha_{g}}\left(\beta_{g}\right)\right)$ intersects $\beta_{g}$ in exactly one point, whence $\operatorname{rk} \operatorname{HF}\left(\psi\left(\beta_{g}\right), \beta_{g}\right)=$ $\iota\left(\beta_{g}, \psi\left(\beta_{g}\right)\right)=1$. Because the above triangle is exact, we deduce

$$
\text { rk } \widehat{\operatorname{HFK}}(Y, K ;-g+1) \leqslant 2 \text {. }
$$

The missing piece of information is $\operatorname{rk} \operatorname{HF}\left(\beta_{g, n}, \psi\left(\beta_{g, n}\right)\right)$. To compute it, observe first that

$$
\psi\left(\beta_{g, n}\right)=t_{\beta_{g-1}} \circ t_{\alpha_{g}} \circ t_{\alpha_{g-1}}\left(\beta_{g, n}\right),
$$

because $\beta_{g, n}=t_{c}^{n}\left(\beta_{g}\right)$ does not intersect any of the curves $\alpha_{i}, \beta_{i}$ for $i \leqslant g-2$. We could, in principle, directly compute the intersection number by studying the curves $\beta_{g, n}$ and $\psi\left(\beta_{g, n}\right)$ on the surface $S$. But Lemma 2 conveniently helps to simplify the calculation. First let us apply it to the curves $(a, b, c)=\left(\beta_{g, n}, \alpha_{g}, \alpha_{g-1}\right)$, which gives

$$
\operatorname{rk} \operatorname{HF}\left(t_{\alpha_{g-1}}\left(\beta_{g, n}\right), \alpha_{g}\right)=\operatorname{rk} \operatorname{HF}\left(\beta_{g, n}, \alpha_{g}\right)=1
$$


because $\alpha_{g-1} \cap \alpha_{g}=\varnothing$ (so that the lower term of the exact triangle has rank 0 ) and $\beta_{g, n}$ and $\alpha_{g}$ intersect in a single point.

Now apply Lemma 2 three times to estimate $\operatorname{rk} \operatorname{HF}\left(\beta_{g, n}, \psi\left(\beta_{g, n}\right)\right)=\operatorname{rk} \operatorname{HF}\left(\psi\left(\beta_{g, n}\right)\right.$, $\left.\beta_{g, n}\right)$ :

1. First apply the lemma to $(a, b, c)=\left(t_{\alpha_{g}} t_{\alpha_{g-1}}\left(\beta_{g, n}\right), \beta_{g, n}, \beta_{g-1}\right)$. Since $\beta_{g-1} \cap \beta_{g, n}$ $=\varnothing$, the lower corner of the exact triangle vanishes and we get:

$$
\operatorname{rk} \operatorname{HF}\left(\psi\left(\beta_{g, n}\right), \beta_{g, n}\right)=\operatorname{rk} \operatorname{HF}\left(t_{\alpha_{g}} \circ t_{\alpha_{g-1}}\left(\beta_{g, n}\right), \beta_{g, n}\right) .
$$

2. Next choose $(a, b, c)=\left(t_{\alpha_{g-1}}\left(\beta_{g, n}\right), \beta_{g, n}, \alpha_{g}\right)$. The lower term in the exact triangle is $\operatorname{HF}\left(\alpha_{g}, t_{\alpha_{g-1}}\left(\beta_{g, n}\right)\right) \otimes \operatorname{HF}\left(\beta_{g, n}, \alpha_{g}\right)$ which has rank 1 by (3.2). The exactness of the triangle implies that:

$$
\operatorname{rkHF}\left(t_{\alpha_{g}} \circ t_{\alpha_{g-1}}\left(\beta_{g, n}\right), \beta_{g, n}\right) \geqslant \operatorname{rkHF}\left(t_{\alpha_{g-1}}\left(\beta_{g, n}\right), \beta_{g, n}\right)-1 .
$$

3. Finally, choose $(a, b, c)=\left(\beta_{g, n}, \beta_{g, n}, \alpha_{g-1}\right)$. The upper right term in the corresponding triangle is $\operatorname{HF}\left(\beta_{g, n}, \beta_{g, n}\right)$ which has rank 2 by equation (2.2). It follows that:

$$
\begin{aligned}
\operatorname{rkHF}\left(t_{\alpha_{g-1}}\left(\beta_{g, n}\right), \beta_{g, n}\right) & \geqslant \operatorname{rk}\left(\operatorname{HF}\left(\alpha_{g-1}, \beta_{g, n}\right) \otimes \operatorname{HF}\left(\beta_{g, n}, \alpha_{g-1}\right)\right)-2 \\
& =\left(\operatorname{rkHF}\left(\alpha_{g-1}, \beta_{g, n}\right)\right)^{2}-2 .
\end{aligned}
$$

Summing up we get

$$
\operatorname{rk} \operatorname{HF}\left(\psi\left(\beta_{g, n}\right), \beta_{g, n}\right) \geqslant\left(\operatorname{rk} \operatorname{HF}\left(\alpha_{g-1}, \beta_{g, n}\right)\right)^{2}-3 .
$$

On the other hand, the computation in Section 2.1 gives $\operatorname{rk} \operatorname{HF}\left(\alpha_{g-1}, \beta_{g, n}\right)=$ $\iota\left(\alpha_{g-1}, \beta_{g, n}\right)=4 n$, so that:

$$
\operatorname{rk} \operatorname{HF}\left(\psi\left(\beta_{g, n}\right), \beta_{g, n}\right) \geqslant 16 n^{2}-3 .
$$

Finally, substituting the last estimation and the one in (3.1) in the rank inequality obtained at the beginning of this section we get:

$$
\begin{aligned}
\operatorname{rk} \widehat{\operatorname{HFK}}\left(S^{3}, K_{g, n} ;-g+1\right) & \geqslant \operatorname{rk} \operatorname{HF}\left(\beta_{g, n}, \psi\left(\beta_{g, n}\right)\right)-\operatorname{rk} \widehat{\operatorname{HFK}}(Y, K ;-g+1) \\
& \geqslant 16 n^{2}-5 .
\end{aligned}
$$

If $n \neq 0$ this quantity is strictly greater than 1 and Theorem 3 implies that $K_{g, n}$ cannot be an L-space knot. This establishes the third property stated in the Theorem. Properties (1) and (2) are proven in [10].

Acknowledgments. The authors would like to thank Michel Boileau and Paolo Ghiggini for many useful discussions. Siddhi Krishna helped to clarify an imprecision in the first version of this article. FM would like to further thank Marco Golla, Beibei Liu and Juanita Pinzón-Caicedo for interesting conversations and helpful remarks. He finally thanks the Max Planck Institute for Mathematics, Bonn, for its support and hospitality, where part of this work was completed. GS thanks the Laboratoire de Mathématiques Nicolas Oresme, Caen, for its support and hospitality. 


\section{REFERENCES}

1. J. Berge, Some knots with surgeries yielding lens spaces (2018), facsimile of an unpublished manuscript from circa 1990, arXiv:1802.09722.

2. M. Boileau, S. Boyer and C. Gordon, Branched covers of quasipositive links and L-spaces, J. Topol. 12 (2019), 536-576, arXiv:1710.07658.

3. D. Eisenbud and W. Neumann, Three-dimensional link theory and invariants of plane curve singularities, Ann. Math. Stud. 110 (1985), Princeton University Press, Princeton, NJ, ISBN: 0-69108380-0; 0-691-08381-9.

4. B. Farb and D. Margalit, A primer on mapping class groups (Princeton University Press, Princeton, NJ, 2012).

5. P. Ghiggini, Knot Floer homology detects genus-one fibred knots, Am. J. Math. 130(5) (2008), 1151-1169, arXiv:math/0603445.

6. J. E. Greene, The lens space realization problem, Ann. Math. (2) 177(2) (2013), 449-511, arXiv: 1010.6257.

7. M. Hedden, On knot Floer homology and cabling: 2, Int. Math. Res. Not. (12) (2009), 2248-2274, arXiv:0806.2172.

8. M. Hedden, Notions of positivity and the Ozsváth-Szabó concordance invariant, J. Knot Theory Ramifications 19(5) (2010), 617-629, arXiv:math/0509499. 299-350.

9. A. Juhász, Floer homology and surface decompositions, Geom. Topol. 12(1) (2008),

10. F. Misev, On families of fibred knots with equal Seifert forms, to appear in Communications in Analysis and Geometry (2017), arXiv:1703.07632.

11. H. R. Morton, Fibred knots with a given Alexander polynomial, in Knots, braids and singularities (Plans-sur-Bex, 1982), Monographs in Mathematics, vol. 31 (European Mathematical Society, Geneva, 1983), 205-222.

12. L. Moser, Elementary surgery along a torus knot, Pacific J. Math. 38(3) (1971), 737-745.

13. Y. Ni, Knot Floer homology detects fibred knots, Invent. Math. 170(3) (2007), 577-608, arXiv:math/0607156.

14. Y. Ni, Erratum: Knot Floer homology detects fibred knots, Invent. Math. 177(1) (2009), 235-238.

15. P. Ozsváth and Z. Szabó, Holomorphic disks and knot invariants, Adv. Math. 186(1) (2004), $58-116$.

16. P. Ozsváth and Z. Szabó, On knot Floer homology and lens space surgeries, Topology 44 (2005) 1281-1300.

17. P. Ozsváth and Z. Szabó, On the Heegaard Floer homology of branched double-covers, $A d v$. Math. 194(1) (2005), 1-33. 2003).

18. J. Rasmussen, Floer homology and knot complements, PhD Thesis (Harvard University,

19. L. Rudolph, Constructions of quasipositive knots and links, I, in Nœuds, Tresses, et Singularités (Weber C., Editor), L'Enseignement Mathématique, vol. 31 (Kundig, Geneva, 1983), 233-246.

20. P. Seidel, A long exact sequence for symplectic Floer cohomology, Topology 42(5) (2003), 1003-1063, arXiv:math/0105186. 